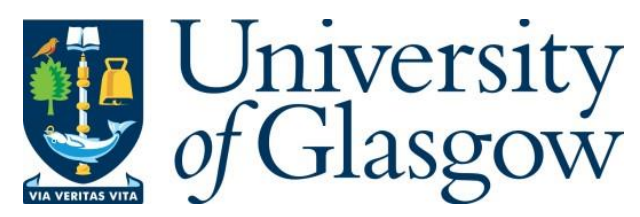

Parreira, P. and Yao, E. (2018) Experimental design laboratories in introductory physics courses: enhancing cognitive tasks and deep conceptual learning. Physics Education, 53, 055012. (doi:10.1088/1361-6552/aacf23)

There may be differences between this version and the published version. You are advised to consult the publisher's version if you wish to cite from it.

http://eprints.gla.ac.uk/164131/

Deposited on: 19 June 2018

Enlighten - Research publications by members of the University of Glasgow http://eprints.gla.ac.uk 


\title{
Experimental Design Laboratories in Introductory Physics Courses: Enhancing Cognitive Tasks and Deep Conceptual Learning
}

\author{
Pedro Parreira \\ SUPA, School of Physics \& Astronomy, University of Glasgow, Glasgow G12 8QQ \\ E-mail: pedromiguel.raimundoparreira@glasgow.ac.uk \\ Eric Yao \\ SUPA, School of Physics \& Astronomy, University of Glasgow, Glasgow G12 8QQ \\ E-mail: eric.yao@glasgow.ac.uk
}

\begin{abstract}
Being able to contextualise and solve complex problems is a highly valued skill in STEM graduates - a skill which we strive to nurture in our students. Since its introduction into undergraduate teaching, laboratory teaching has been used to consolidate students conceptual understanding, develop their practical skills and inculcate an evidence based problem solving approach. Much work has been done to achieve these goals with varying degrees of success. Here we present an alternative to the regular introductory level physics laboratory experiment which enhances students learning by focusing on problem solving rather than simply following detailed instructions. Working in small groups, students were able achieve the aims of the experiment through self and peerinstruction. Similar experiments can be easily and cost effectively implemented in any standard secondary school and undergraduate teaching laboratory. These can be adjusted to target the development of a wide range of specific skill sets as well as deepen students understanding of different physics principles and concepts. Our approach will enable the teaching laboratory to truly fulfil the function with which it was originally conceived.
\end{abstract}

PACS numbers: $00.00,20.00,42.10$

Keywords: cognitive tasks, introductory physics, labs, deep learning, conceptual learning.

\section{Introduction}

Undergraduate introductory laboratory sessions are, to many physicists, an integral and essential component of the education in physics [1] and are normally a requirement for the external accreditation of the course. Until recently, little research had been 
done regarding the benefits of introductory physics laboratories to students. Not long ago, Wieman et al. suggested that there is hardly any difference between students that took labs and those who did not regarding their performance in experimental related questions in exams. Furthermore, it has also been suggested that the cognitive benefits involved in the aforementioned labs is very likely limited because of the way the experimental challenge is presented. Introductory physics laboratory work in elite higher education institutions is often portrayed as 'cookbook', where students are asked to follow a rather well detailed set of instructions. Additionally, labs often make use of fully-assembled experimental equipment which further contributes to diminish student motivation $[2,3]$. Limited benefits are of particularly importance because of the high resources required for introductory laboratory classes, specifically when it comes to the allocation of teaching space and staff. Therefore, designing lab activities aimed at increasing student motivation towards developing their experimental skills is of critical importance. One way to overcome a 'cookbook' type laboratory session in introductory physics courses is to emphasise the experimental design aspect of the lab by focusing on group-based problem solving. In other words, students are asked to develop an experimental procedure such that a particular physical property or concept can be verified or demonstrated, as opposed to following the conventional and very detailed laboratory script. Students working in small collaborative groups learn what they need to know in order to solve a given problem, which is the basic framework of problem-based learning [4]. Additionally, working in small groups enhances discussions that encourages a deeper approach to learning by avoiding memorisation of discreet and often unrelated facts [5]. In fact experimental design labs are intended to enhance student learning by having them actively construct their own meanings which are consistent with their own prior ideas as well as by the social process of communicating with their peers [5]. If the student's prior knowledge and the new disciplinary knowledge are not connected and intertwined, learning of scientific concepts is reduced to the memorisation of facts $[5,6]$. Interestingly, group-based problem solving activities that are part of the experimental design lab are often employed or mentioned within the flipped classroom framework when discussing alternative pedagogic techniques [7, 8]. One implication of such an approach is that experimental design laboratory sessions will likely need to be longer than regular sessions. Such demand has to do with the fact that in experimental design laboratories students are more focused on planning, reflecting and making predictions rather than following a set of instructions. That is to say, students are encouraged to spend time understanding and internalising concepts as well as planning on what to invest their time on during the session. Experimental design can also be regarded as an integrative activity. In other words, when designing experiments students will often deal with several branches of physics: from electronic circuits to thermal physics and from classical mechanics to optics, while at the same time developing their programming and experimental data analysis skills. Therefore, experimental design labs are better alternatives to regular labs because they promote a deeper conceptual learning and enhanced peer interaction in introductory physics courses. 


\section{Setting}

One key aspect of the experimental design laboratories relates to the fact that students are instructed to discuss in small groups all of the stages of their experimental challenge. Such group discussions will often deal with identifying and choosing the equipment, developing the knowledge on how to assemble it, consider how to gather the data and decide how to analyse and present it adequately afterwards. Not surprisingly, the experimental design lab consists of two main sessions: the first session deals with familiarisation with the equipment and planning whereas the second focuses on dealing with the experimental challenge itself. Before the beginning of the experimental sessions, students are presented with the challenge and introduced to the equipment they will be using. Students are asked to perform some background reading related to the experimental challenge. A pre-lab assessment is used to encourage active engagement with the reading material. At the start of the session, students are shown examples of experiments where similar equipment is used in more advanced research. The next step is to suggest to students that they should familiarise themselves with the equipment. Students are given a set of demonstration exercises which utilises different components of the equipment. Some simple tasks are set which require the students to understand and then modify or adjust the equipment. By the end of the first lab session, students are reminded of the single straightforward challenge to be addressed during the next session. Examples of possible challenges involves developing an experimental procedure to determine a physical constant (say $g$, for instance), build a data-logger and/or perform an instrument calibration, amongst several other possibilities. The second lab session is then spent on assembling the required electronic circuits, constructing the apparatus, gathering and analysing data. By the end of the second lab session, all groups are asked to compare their findings, in an effort to understand the differences between their experimental results and possible reasons for the discrepancies. It is worth pointing out that during the first session, students are encouraged to ask and discuss with staff as much as they feel necessary. However, they are advised that during the second session they should perform their experimental activities autonomously. Finally, the experimental design lab ends with the submission of a brief two page report where students are asked to present the data as well as a brief description of their experimental procedure. In summary, our approach to laboratory teaching focuses on goal-oriented and multifaceted group work aimed at enhancing deeper conceptual understanding.

\section{Example of an Experimental Design Lab: Building a Data-logger with an Arduino}

Here we report on the development of an autonomous data-logger and on the calibration of a commercial thermistor using a calibrated thermocouple, all interfaced with a microcontroller board connected to a PC. Arduino boards were used because of its simplicity and low cost, but perhaps more importantly, because of the abundant freely accessible 
online resources. It is therefore no surprise that Arduino boards have been used throughout research groups in engineering and physics having recently made their way into introductory physics labs [9-13]. For the first part of the lab, students were told to investigate how to connect the Arduino board to a PC as well as investigating the basis of programming. It is assumed that the majority of the cohort had no previous knowledge of coding, at least not proficiently. No tutorial or lecture was provided regarding coding, instead students were asked to explore at a few simple built-in examples included in the Arduino interface library and learn by themselves as a group. A good example of a builtin routine is the 'Blink' example program that switches a built-in LED on and off every second. By looking at the functions within the example program, namely DigitalWrite HIGH, DigitalWrite LOW and Delay functions, students can understand the underlying mechanisms of a simple program. As for the calibration exercise itself, the thermistor was connected to the Arduino board with a voltage divider circuit using the analog input pins i.e. the voltage from the thermistor was converted using the built in 10-bit analogto-digital converter within the Arduino board. However, before attempting to use the thermistors, students were encouraged to assemble a voltage divider circuit where one of the resistors is a potentiometer, as illustrated in figure 1a. Fitting a potentiometer to the circuit allows for students to appreciate how a variable resistor impacts on the voltage read by the Arduino board.

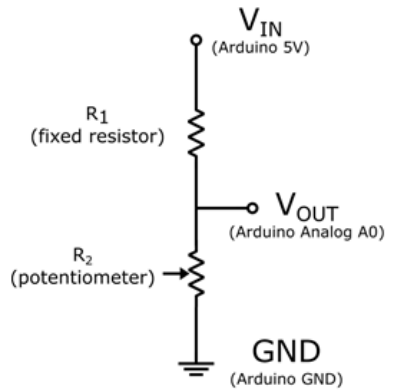

a)

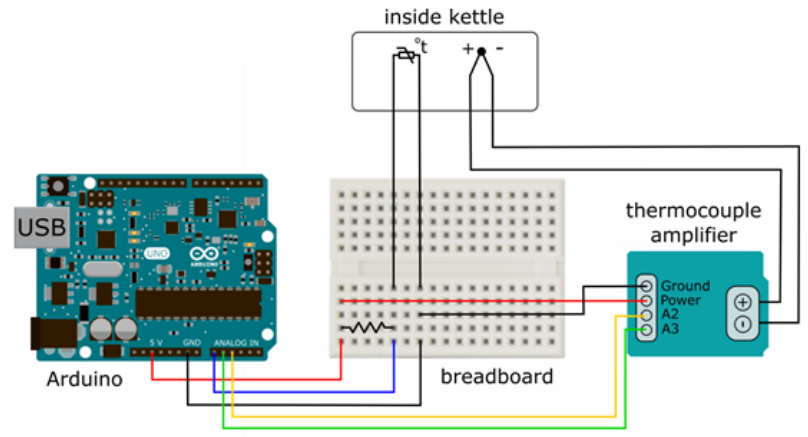

b)

Figure 1: a) Circuit diagram of the voltage divider used, showing where the Arduino pins are connected to, and b) simple schematic illustrating how the experimental apparatus developed by the students is set up.

By varying the resistance of the potentiometer students can observe how the change in resistance affects the voltage read by the Arduino analog-to-digital converter, which is numerically or graphically represented within the Arduino PC interface. The thermocouple used for the calibration of the thermistor was connected to the Arduino board using two analog pins, one for the cold and one for the hot junction. The first part of the lab ends when all group members agreed that they have gathered enough knowledge to allow for them to execute the calibration experiment autonomously in the following session. The second session started with students assembling the experimental apparatus and beginning with preliminary testing mainly to assess their 
programs. The consensus amongst the majority of the groups about how the calibration procedure should be undertaken was the following: the thermistor and the thermocouple were attached to one another and both interfaced with the Arduino board which was connected to a PC, as depicted in figure 1b. Students also checked the default calibration of the thermocouple by measuring the temperature of an ice melt and of boiling water. Both sensors were then placed inside a kettle which was filled half way with an ice melt. Soon after data logging was initiated and a few seconds later the kettle was turned ON, melting the ice and heating the water. Once the water starts to boil, the system was allowed to run for a few seconds before turning the kettle OFF. A typical set of results, obtained by students, is presented in figures $2 \mathrm{a}$ and $2 \mathrm{~b}$. Figure $2 \mathrm{a}$ is obtained when the temperature values from the calibrated thermocouple are plotted as a function of time. Note that the elapsed time is also given by the Arduino code written by the students. Figure $2 \mathrm{~b}$ is obtained by plotting the analog-to-digital (ADC) converted values from the thermistor (via the voltage divider circuit, illustrated in figure 1a) versus the temperature read by the calibrated thermocouple. The fitting functions used were not the same for all groups, but it was shown that a third order polynomial or logarithmic functions work best. Once the parameters were determined, students incorporated the fitting function into the Arduino program so that the thermistor outputs a temperature value instead of a voltage value via the ADC.

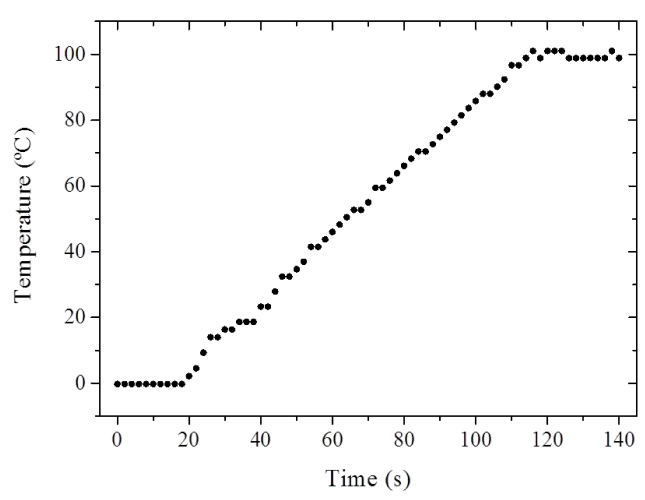

a)

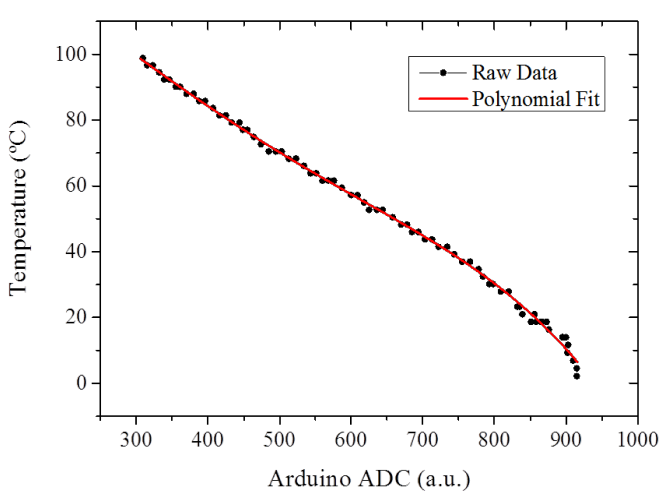

b)

Figure 2: a) Raw data from the calibrated thermocouple plotted as a function of elapsed time, and b) example of a calibration plot obtained by students (Arduino ADC signal as a function of temperature measured by the thermocouple). The discreet temperature steps (seen in both plots) are due to the resolution of the thermocouple connected to the Arduino, which is in the range of $2{ }^{\circ} \mathrm{C}$. For the plot in b) a third order polynomial was used for the fitting.

The final experimental stage was to ask all groups of students to measure the temperature of a control substance provided by staff using the thermistor circuit only in order to appreciate how their results vary. The temperature measured by their setups is compared to the temperature measured with three standard commercial thermocouples which have been used by the students in previous regular experiments. Within the groups, students were instructed to draw conclusions that explain the 
variability observed amongst all groups. Finally, students were instructed to summarise their findings in a two page report comprising the calibration plot, the code used to acquire the data and how their measured temperature compares to the real temperature of the control substance.

\section{Metodology}

We introduced the experimental design laboratory to level 1 physics classes over a period of three years. Typical class size is 280 and the cohort is divided in groups of five students. The experimental design laboratory takes 2 three-hour sessions over a period of two weeks. Students were asked to submit their feedback in groups together with the lab report during the 2016-2017 academic year, so the total number of questionnaires is 51. The questions were: Question 1 - What are the benefits and drawbacks of the experimental design project compared to the standard laboratory experiments you have performed over the academic year? and Question 2 - Aspects of the experimental design project that can be improved? Due to the open nature of the questions, responses from students were grouped in categories. Completion of the questionnaire was voluntary and the number of answers represented $93 \%$ of the class.

\section{Results}

The following discussion details the results obtained for the two questions presented in the previous section. Analysis was carried out with frequency data tabulated on a spreadsheet. Table 1 summarises the results from the first question.

Table 1: Summary of results obtained from question 1. 'What are the benefits and drawbacks of the experimental design project compared to the standard laboratory experiments you have performed over the academic year?' Numbers in percentage. Based on 51 answers.

\begin{tabular}{ll}
\hline Benefits & $\%$ \\
Not following instructions & 80 \\
Working in small groups & 67 \\
Using Arduino & 51 \\
Having more time & 20 \\
Meeting new people & 12 \\
\hline Drawbacks & $\%$ \\
Not based on lectures/physics & 29 \\
Groups too big & 24 \\
Difficult to manage teamwork & 8 \\
Not enough time & 4 \\
Two sessions & 2 \\
\hline
\end{tabular}

Regarding question 1, it should be pointed out that not all groups stated a drawback, which contrast with the several benefits stated on each questionnaire. In fact, there were 134 comments on benefits whereas drawback only gathered 34, which at the very least, 
attests to the enthusiasm of the students with the experimental design lab. Given the open nature of the questions, student's comments were grouped into three categories: (1) benefit of not having a conventional lab script, (2) importance of teamwork and (3) the advantages of using Arduino boards. The following comments summarise the student's views on (1) not having to follow a detailed lab script:

- This experiment was much more interesting, engaging and required some creative work rather than just following given instructions.

- Standard lab experiments are quite mechanical (rigid in terms of following lab script). It was nice to be given the autonomy to approach the task creatively.

- More trial and error involved in this experimental design project compared to just following the manual and answering questions, this means we get the chance to mess around more freely with equipment and learn by doing.

Not having a detailed lab script gives the students the opportunity to assemble the lab equipment and actually do the experiment themselves. It is the sense of discovery, achievement and reward that most motivated the students and is the biggest advantage if goal oriented learning. The following sentences summarise their feedback regarding (2) having to work in small groups:

- We found the teamwork aspect of the experiment beneficial as it encouraged us to support each other, to find everyones strengths and weaknesses and work together to best support each other. We were also able to learn from each other, and work on our communication skills.

- Being able to work in groups allowed us to gain knowledge from each other and also allows us to explain concepts to each other in a different way from lecturers which can be very helpful if a lecturers way of explaining things is unsuited to your way of learning.

- First of all, it allowed us to learn how to organise with different people. That means skills to understand other group members points of views have been developed and communication skills have been improved.

Group work is another the key aspect of the experimental design labs. In fact, it is the group work aspect of the lab that is critical in identifying student's strengths and weaknesses, encouraging them to engage in fruitful discussions. A very clear additional benefit is the development of student's communication skills. The following comments illustrate the student perception regarding (3) the use of Arduino boards:

- The addition of parts of coding and computing are a great way to introduce students to the importance of computing science in modern physics. It presents a challenge as the regular assembly of apparatus is very different to the more mentally demanding and accurate requirements of operating the Arduino. 
- Independent learning - in reference to the coding of the Arduino programme - by use of Internet research.

- It was satisfying to eventually figure out how the Arduino boards worked, when they seemed quite challenging to understand at the beginning.

Arduino boards are particularly useful because, unlike more powerful alternatives that mostly resemble a computer, Arduinos require a certain degree of circuit assembly. Additionally, students must write simple programs within a non-graphical interface, which is an excellent method to introduce them to programming within the context of physics. Perhaps most importantly, the amount of resources available on the web ensures that students can look up information autonomously, which is the intention of this type of approach. Another characteristic of the experimental design lab is having more time. By removing the time constraint students develop their planning skills, but more importantly, it allows them to execute more complex experiments without detailed instructions. Giving time for students to incorporate their new discoveries in a deep and consistent way, so that they can improve on them later, should be one of the objectives of all laboratory sessions. In regards to the drawbacks, most of the concerns raise related to the lack of physics in the experimental design when compared to regular labs, as can be seen from the bottom part of table 1 . As a matter of fact, the few comments students made that were not seemingly positive have to do with the lack of alignment between the lab and the lecture material. The next sentences serve as an example:

- The task itself contained very little physics, or at least, our physics knowledge was rarely tested.

- One drawback to this experiment compared to previous experiments is that it was not tied in with the lectures. This meant that we didn't have much previous knowledge of the experiment, and were unable to put the theory that we have learnt into a practical situation.

- It was quite disjointed from the lecture material.

It is clear that there are links between the 'experimental design' challenge and the material covered in the lectures. One good example of a link between content covered extensively in the lectures and revisited in labs is Ohm's Law - employed when assembling the Arduino data-logger circuit. Another example is the use of an ice melt and boiling water where the concept of Latent Heat is revisited. Critically, it is the concept of calibration that is most appealing, as all instruments used in any physics labs require a calibration. In addition to the aforementioned comments, there were general lab improvements noticed by the teaching and support staff. For instance, having to write simple programs is a key requirement of the experimental design lab and more importantly, it was shown that students can learn how to code by themselves using simple examples. Another, perhaps less obvious benefit, is related 
to student's interaction with lab demonstrators. It was noticed that student's valued the demonstrator's feedback much more when compared with regular labs even if the lab demonstrators present during the experimental design labs were the same. Also, students were clearly more engaged and motivated throughout the two lab sessions, which was very noticeable and indicative that possibly the experimental design lab could be implemented on additional sessions within the academic term. There are, however, a few topics where the labs can be further developed. Table 2 summarises the results obtained for question 2 , where the students were asked to state the aspects of the lab that could be improved.

Table 2: Summary of results obtained from question 2. 'Aspects of this design project that can be improved?' Numbers in percentage. Based on 51 answers.

\begin{tabular}{ll}
\hline Improvements & $\%$ \\
Tutorial on programming & 63 \\
Fix temperamental gear & 20 \\
Clearer objective & 18 \\
\hline
\end{tabular}

Clearly, introducing a tutorial on programming prior to the lab is the most popular suggestion, and as such, it is where the discussion will focus on. It is interesting that all groups mastered the required programming skills without a tutorial. In fact, although the programming requirements of the lab were a challenge, it did not seems to impede any student group from achieving the calibration task. Moreover, been exposed to coding and computing was referred by the same cohort as being beneficial. Instead, the feedback regarding the lack of a programming tutorial could perhaps be the cause preventing the lab tasks being linked to the lecture material - as students are more focused on the coding/computing aspects of the challenge. Interestingly, it points to the idea that there is a fine balance between the time spent on dealing with the equipment and on specific physics related tasks. On way to mitigate such an issue could be to expand on the experimental design laboratory by having students use their temperature data-loggers in physics-specific contexts, like for instance, determining the specific heat capacity of substances.

\section{Conclusions}

An alternative to regular laboratory session was presented under the form of an experimental design lab. Results suggest that student perception regarding the alternative labs was very positive, which may explain their overall better performance when compared to regular labs. Furthermore, it was demonstrated how the use of generic equipment, under the form of Arduino boards, has advantages when compared to conventional lab teaching. In addition, the change from a cookbook-like laboratories to sessions where the experimental design is emphasised allows students to solve more 
complex problems instead of merely following instructions. It also allows students to collaborate in the planning and assembling of the apparatus, including by constructing circuits and writing simple programs. Finally, experimental design labs are cost effective and can be adjusted to target the development of a wide range of skill sets in other STEM disciplines in standard secondary schools or in higher education.

\section{References}

[1] Carl Wieman, N G Holmes, and Carl Wieman. "Measuring the impact of an instructional laboratory on the learning of introductory physics". In: American Journal of Physics 83.11 (Nov. 2015), pp. 972-978. DOI: 10.1119/1.4931717.

[2] Carl Wieman. "Comparative Cognitive Task Analyses of Experimental Science and Instructional Laboratory Courses". In: The Physics Teacher 53.6 (Sept. 2015), pp. 349-351. DOI: 10.1119/1.4928349.

[3] Benjamin M Zwickl, Noah Finkelstein, and H J Lewandowski. "Incorporating learning goals about modeling into an upper-division physics laboratory experiment". In: American Journal of Physics J. Phys. J. Phys. J. Phys 82.101 (Sept. 2014), pp. 4789659-485. DOI: 10.1119/1.4875924. arXiv: arXiv: 1301 . $4414 \mathrm{v} 2$.

[4] Cindy E. Hmelo-silver. "Problem-Based Learning: What and How Do Students Learn?" In: Educational Psychology Review 16.3 (Sept. 2004), pp. 235-266. DOI: 10.1023/B:EDPR.0000034022.16470.f3.

[5] Christine Chin and David E. Brown. "Learning in Science: A Comparison of Deep and Surface Approaches". In: Journal of Research in Science Teaching 37.2 (Feb. 2000), pp. 109-138. DOI: 10.1002/(SICI) 1098-2736(200002) $37: 2<109:$ :AIDTEA3>3.0.CO;2-7.

[6] Kathleen J Roth, BF Jones, and L Idol. "Developing meaningful conceptual understanding in science". In: Dimensions of thinking and cognitive instruction (1990), pp. 139-175.

[7] Jacob Bishop and Matthew Verleger. "The Flipped Classroom : A Survey of the Research". In: Proccedings of the Annual Conference of the American Society for Engineering Education (2013), p. 6219.

[8] Eric Mazur. "EDUCATION: Farewell, Lecture?" In: Science 323.5910 (Jan. 2009), pp. 50-51. DOI: 10.1126/science.1168927.

[9] Calin Galeriu. "An Arduino-Controlled Photogate". In: The Physics Teacher 51.3 (2013), p. 156. DOI: 10.1119/1.4792011.

[10] Calin Galeriu, Scott Edwards, and Geoffrey Esper. "An Arduino Investigation of Simple Harmonic Motion". In: The Physics Teacher 52.3 (2014), pp. 157-159. DOI: 10.1119/1.4865518. 
[11] Francisco Vera, Rodrigo Rivera, and Manuel Ortiz. "A simple experiment to measure the inverse square law of light in daylight conditions". In: European Journal of Physics 35.1 (Jan. 2014), p. 015015. DOI: 10.1088/0143-0807/35/1/ 015015.

[12] K Zachariadou, K Yiasemides, and N Trougkakos. "A low-cost computercontrolled Arduino-based educational laboratory system for teaching the fundamentals of photovoltaic cells". In: European Journal of Physics 33.6 (Nov. 2012), pp. 1599-1610. DOI: 10.1088/0143-0807/33/6/1599.

[13] Stepanka Kubinova and Jan Slegr. "Physics demonstrations with the Arduino board". In: Physics Education 50.4 (2015), p. 472. DOI: 10.1088/0031-9120/50/ $4 / 472$. 